\title{
Numerical Methods for the Hydrodynamic Device Model: Subsonic Flow
}

\author{
CARL L. GARDNER, JOSEPH W. JEROME, AND DONALD J. ROSE
}

\begin{abstract}
An introduction to the hydrodynamic model for semiconductor devices is presented. Special attention is paid to classifying the hydrodynamic PDE's and analyzing their nonlinear wave structure. Numerical simulations of the ballistic diode using the hydrodynamic device model are presented, as an illustrative elliptic problem. The importance of nonlinear block iterative methods is emphasized. Arguments for existence of solutions and convergence of numerical methods are given for the case of subsonic electron flow.
\end{abstract}

\section{INTRODUCTION}

$\mathrm{T}$ HE hydrodynamic model [1], [2] plays an important role in simulating the behavior of charge carriers in submicron semiconductor devices, since it exhibits velocity overshoot [3]-[5] and ballistic effects [6]-[8] missing in the drift-diffusion model. The hydrodynamic equations consist of a set of nonlinear conservation laws for particle number, momentum, and energy, plus Poisson's equation for the electric potential. The nonlinear conservation laws are just the Euler equations [9] $(\gamma=5 / 3)$ of gas dynamics for a gas of charged particles in an electric field, with the addition of a heat conduction term. Thus the hydrodynamic model PDE's have hyperbolic, parabolic, and elliptic modes.

Imagine a gas of electrons flowing in a semiconductor. This gas has a soundspeed, and the electron flow may be either subsonic or supersonic. In the case of supersonic flow, we must allow the possibility of electron shock waves in semiconductor devices. This will be the subject of a subsequent paper [10].

In this investigation we focus our attention on appropriate numerical methods for both time-dependent and steady-state simulations when the electron flow is subsonic. The 1-D steady-state equations will then be elliptic.

In Section II, we discuss the hydrodynamic PDE's and analyze their nonlinear wave structure. In Section III, we

Manuscript received August 5, 1988; revised December 11. 1988. C. L. Gardner was supported in part by the Office of Naval Research under Contract N00014-85-K-0487 and by the Microelectronics Center of North Carolina. J. W. Jerome was supported in part by the National Science Foundation under Grant DMS-872-1742. D. J. Rose was supported in part by the Office of Naval Research under Contract N00014-85-K-0487, by the Microelectronics Center of North Carolina, and by AT\&T Bell Laboratories. The review of this paper was recommended by Guest Editor M. Pinto.

C. L. Gardner and D. J. Rose are with the Computer Science and Mathematics Department, Duke University, Durham, NC 27706.

J. W. Jerome is with the Department of Mathematics, Northwestern University, Evanston, IL 60208.

JEEE Log Number 8826358 . present the results of steady-state simulations of a 1-D ballistic diode using these methods, and compare our results with those of Odeh, Rudan, and White [11].

For the model ballistic diode problem, the effects of holes may be neglected. The hydrodynamic model in one dimension then consists of three nonlinear conservation laws (for electron number, momentum, and energy), plus Poisson's equation for the electric potential.

Our numerical methods are inspired by computational fluid dynamics: as state variables we use the electron density, velocity, and temperature, plus the electric potential. We use a damped Newton method [12] to linearize the device equations. We then compare direct and iterative methods for solving these linearized equations. In the iterative method case, we discuss how to optimize the number of inner iterative loops vs. outer Newton loops.

In Section IV, arguments are given for the existence of solutions and for the convergence of Newton's method when the electron flow is subsonic. These arguments justify the use of the numerical methods described in Section III.

\section{The Hydrodynamic Model}

\section{The Hydrodynamic Model Equations}

A fairly general and rigorous set of transport equations for device simulation has been derived by Blotekjaer [1] from the Boltzmann equation:

$$
\begin{gathered}
\frac{\partial n}{\partial t}+\nabla \cdot(n \boldsymbol{v})=\left(\frac{\partial n}{\partial t}\right)_{c} \\
\frac{\partial \boldsymbol{p}}{\partial t}+\boldsymbol{v} \nabla \cdot \boldsymbol{p}+\boldsymbol{p} \cdot \nabla \boldsymbol{v}=-e n \boldsymbol{E}-\nabla(n T)+\left(\frac{\partial \boldsymbol{p}}{\partial t}\right)_{c} \\
\frac{\partial W}{\partial t}+\nabla \cdot(\boldsymbol{v} W) \\
=-e n \boldsymbol{v} \cdot \boldsymbol{E}-\nabla \cdot(\boldsymbol{v} n T)-\nabla \cdot \boldsymbol{q}+\left(\frac{\partial W}{\partial t}\right)_{c}
\end{gathered}
$$

where $n$ is the electron density, $v$ is the velocity, $p$ is the momentum density, $e(>0)$ is the electronic charge, $\boldsymbol{E}$ is the electric field, $T$ is the temperature in energy units (Boltzmann's constant $k$ has been set to 1 ), $W$ is the en- 
ergy density, $q$ is the heat flow, and the subscript $c$ represents collision terms.

We will assume that

$$
q=-\kappa \nabla T
$$

and that the energy bands are parabolic:

$$
\begin{aligned}
p & =m n v \\
W & =\frac{3}{2} n T+\frac{m}{2} n v^{2}
\end{aligned}
$$

where $m$ is the effective electron mass.

In addition to the transport equations (1)-(3), we have Poisson's equation for the electric field

$$
\begin{aligned}
\boldsymbol{E} & =-\nabla \phi \\
\nabla \cdot(\epsilon \nabla \phi) & =-e\left(N_{D}-N_{A}-n\right)
\end{aligned}
$$

where $N_{D}$ is the density of donors and $N_{A}$ is the density of acceptors. Equations (1)-(7) determine the variables $n, p$, $W$, and $\phi$.

The collision terms in (1)-(3) may be expressed in terms of phenomeno-logical relaxation times. This approximation should be valid for devices with active regions $\geq 0.1$ $\mu \mathrm{m}$, since at this length scale simulations of the hydrodynamic model are in good agreement with Monte Carlo simulations of the Boltzmann equation [11], [14]. At some stage though, as active device lengths become shorter and shorter, the hydrodynamic approximation will become invalid, and the full Boltzmann equation must be simulated.

Equations (1)-(3) may be recast [1] in terms of the variables $n, v, T$, and $\phi$ :

$$
\begin{aligned}
\frac{\partial n}{\partial t}+\nabla \cdot(n \boldsymbol{v})= & \left(\frac{\partial n}{\partial t}\right)_{c} \\
\frac{\partial \boldsymbol{v}}{\partial t}+\boldsymbol{v} \cdot \nabla \boldsymbol{v}= & -\frac{e}{m} \boldsymbol{E}-\frac{1}{m n} \nabla(n T)+\left(\frac{\partial \boldsymbol{v}}{\partial t}\right)_{c} \\
\frac{\partial T}{\partial t}+v \cdot \nabla T= & -\frac{2}{3}(\nabla \cdot v) T \\
& +\frac{2}{3 n} \nabla(\kappa \nabla T)+\left(\frac{\partial T}{\partial t}\right)_{c}
\end{aligned}
$$

where

$$
\begin{aligned}
\left(\frac{\partial v}{\partial t}\right)_{c}= & \frac{1}{m n}\left(\frac{\partial p}{\partial t}\right)_{c}-\frac{\boldsymbol{v}}{n}\left(\frac{\partial n}{\partial t}\right)_{c} \\
\frac{3}{2}\left(\frac{\partial T}{\partial t}\right)_{c}= & \frac{1}{n}\left(\frac{\partial W}{\partial t}\right)_{c}-\frac{\boldsymbol{v}}{n}\left(\frac{\partial \boldsymbol{p}}{\partial t}\right)_{c} \\
& +\left(\frac{\frac{m}{2} v^{2}-\frac{3}{2} T}{n}\right)\left(\frac{\partial n}{\partial t}\right)_{c} .
\end{aligned}
$$

This form is useful for classifying the equations and calculating the characteristic speeds.

\section{Nonlinear Wave Structure}

If $\kappa$ is set to zero in (4), then (1)-(3) are the Euler equations with source terms due to the collision terms and the electric field. (The polytropic gas constant $\gamma=5 / 3$, as is appropriate for a monatomic gas.) These equations then are hyperbolic, with five nonlinear waves [9], [13]:

\begin{tabular}{|l|c|c|}
\hline $\begin{array}{c}\text { nonlinear } \\
\text { wave }\end{array}$ & $\begin{array}{c}\text { characteristic } \\
\text { speed }\end{array}$ & $\begin{array}{c}\text { discontinuous } \\
\text { variables }\end{array}$ \\
\hline 2 shocks & $\lambda_{ \pm}=v \pm c$ & $v, n, T$ \\
\hline 3 contacts & $\lambda_{0}=v$ & $v_{T}, T$ \\
\hline
\end{tabular}

$v$ is the particle velocity normal to the wave, and $v_{T}$ is the particle velocity tangential to the wave. The soundspeed $c=\sqrt{\gamma T / m}$.

If now we let $\kappa$ be nonzero, (1)-(3) become hyperbolic (4 modes) plus parabolic ( 1 mode). There are now only four nonlinear hyperbolic waves:

\begin{tabular}{|l|c|c|}
\hline $\begin{array}{c}\text { nonlinear } \\
\text { wave }\end{array}$ & $\begin{array}{c}\text { characteristic } \\
\text { speed }\end{array}$ & $\begin{array}{c}\text { discontinuous } \\
\text { variables }\end{array}$ \\
\hline 2 shocks & $\lambda_{ \pm}=v \pm c$ & $v, n$ \\
\hline 2 contacts & $\lambda_{0}=v$ & $v_{T}$ \\
\hline
\end{tabular}

The contact wave corresponding to a jump in $T$ has disappeared due to the $\nabla \cdot(\kappa \nabla T)$ term in $(10)$, and the soundspeed $c=\sqrt{T / m}$. For $T=\alpha T_{0}$ and $T_{0}=300 \mathrm{~K}, c$ $=\sqrt{\alpha} 1.3 \times 10^{7} \mathrm{~cm} / \mathrm{s}$.

These results are derived and analyzed in more detail in [10].

\section{Numerical Solution of the Hydrodynamic Equations for the Ballistic Diode}

\section{The Ballistic Diode}

As a model 1-D steady-state problem, we simulate the flow of electrons in a submicron $n^{+}-n-n^{+}$silicon diode. This device models the $n^{+}-n-n^{+}$channel in a MOSFET, and clearly exhibits hot carrier effects at submicron seales. The diode consists of a $0.1 \mu \mathrm{m} \mathrm{n}^{+}$-region, followed by a $0.4 \mu \mathrm{m} \mathrm{n}$ region, and ending with a $0.1 \mu \mathrm{m}$ $\mathrm{n}^{+}$region. In the $\mathrm{n}^{+}$region, the doping density $N=5 \times$ $10^{17} \mathrm{~cm}^{-3}$, while in the $\mathrm{n}$ region $N=2 \times 10^{15} \mathrm{~cm}^{-3}$. The ambient device temperature $T_{0}=300 \mathrm{~K}=0.025 \mathrm{eV}$.

Our parameters are identical with those of Odeh, $\mathrm{Ru}$ dan, and White [11], so that we can compare the two sets of computations. Their computations also agree with Monte Carlo simulations [11], [14] of the same device.

\section{Relaxation Time and Heat Conduction Models}

Since the effects of holes may be neglected for the ballistic diode problem,

$$
\left(\frac{\partial n}{\partial t}\right)_{c}=0
$$


Following Baccarani and Wordeman [5], we take

$$
\begin{aligned}
\left(\frac{\partial \boldsymbol{p}}{\partial t}\right)_{c} & =\frac{-\boldsymbol{p}}{\tau_{p}} \\
\tau_{p} & =m \frac{\mu_{n 0}}{e} \frac{T_{0}}{T} \\
\left(\frac{\partial W}{\partial t}\right)_{c} & =\frac{-\left(W-\frac{3}{2} n T_{0}\right)}{\tau_{w}} \\
\tau_{n} & =\frac{m}{2} \frac{\mu_{n 0}}{e} \frac{T_{0}}{T}+\frac{3}{2} \frac{\mu_{n 0}}{e v_{s}^{2}} \frac{T T_{0}}{T+T_{0}} \\
\kappa & =\kappa_{0} \frac{\mu_{n 0}}{e} n T_{0}, \quad \kappa_{0}=\frac{3}{2}
\end{aligned}
$$

where $\mu_{n 0}$ is the low-field electron mobility and $v_{s}$ is the saturation velocity.

\section{The 1-D Steady-State Equations}

The 1-D steady-state hydrodynamic equations take the following form:

$$
\begin{aligned}
f_{n}= & \frac{d}{d x}(n v)=0 \\
f_{v}= & v \frac{d v}{d x}-\frac{e}{m} \frac{d \phi}{d x}+\frac{1}{m n} \frac{d}{d x}(n T)+\frac{v}{\tau_{p}}=0 \\
f_{T}= & \frac{3}{2} v \frac{d T}{d x}+T \frac{d v}{d x}-\frac{1}{n} \frac{d}{d x}\left(\kappa \frac{d T}{d x}\right) \\
& +\frac{\frac{m}{2} v^{2}+\frac{3}{2}\left(T-T_{0}\right)}{\tau_{w}}-\frac{m v^{2}}{\tau_{p}}=0 \\
f_{\phi}= & \epsilon \frac{d^{2} \phi}{d x^{2}}+e(N-n)=0 .
\end{aligned}
$$

In [2] and [11], the two first-order equations (19) and (20) for $n$ and $v$ are eventually combined into a single second-order equation for $n$, where the nonlinear term $v(d v / d x)$ is linearized about a previous iterate. In the 1-D steady-state problem, this method produces an elliptic equation for $n$, and is justified only as long as the electron flow is everywhere subsonic. In the spirit of computational fluid dynamics, we prefer to solve the full set of conservation laws. Our method then can be extended to the case where the electron flow may become supersonic.

The simplest boundary conditions are $n=N$ and $T=$ $T_{0}$ at $x_{\min }$ and $x_{\max }$, and

$$
\begin{aligned}
& e \phi\left(x_{\min }\right)=T \ln \left(n / n_{e}\right) \\
& e \phi\left(x_{\max }\right)=T \ln \left(n / n_{e}\right)+e V
\end{aligned}
$$

where $V$ is the bias across the diode, and $n_{e}$ is the intrinsic electron concentration.
Physical Parameters: In silicon, the effective electron mass $m=0.26 m_{e}$, where $m_{e}$ is the electron mass, $\epsilon=$ 11.7 and $n_{e}=1.4 \times 10^{10} \mathrm{~cm}^{-3}$. Following [5], we take $\mu_{n 0}=1400 \mathrm{~cm}^{2} / \mathrm{s} \cdot \mathrm{V}$ and $v_{s}=10^{7} \mathrm{~cm} / \mathrm{s}$.

\section{Discretization}

$n, T$, and $\phi$ are defined at the grid points $i=0,1$, $\cdots, N-1, N$. The boundary conditions specify $n, T$, $\phi$ at $i=0$ and $i=N . v$ is defined at the midpoints of the elements $l_{i}(i=1, \cdots, N)$ connecting grid points $i-$ 1 and $i$.

Equations (19), (21), and (22) are enforced at the interior grid points $i=1, \cdots, N-1$, while $(20)$ is enforced at the midpoints of the elements $l_{i}, i=1, \cdots$, $N$.

The discrete equations are then derived by using the 1-D form of the box method (see, e.g., [15]) and central differences.

We use Newton's method to linearize (19)-(22). The resulting operator equation

$$
J\left[\begin{array}{l}
\delta n \\
\delta v \\
\delta T \\
\delta \phi
\end{array}\right]=-\left[\begin{array}{l}
f_{n} \\
f_{\ell} \\
f_{T} \\
f_{\phi}
\end{array}\right]=-f
$$

where $J$ is the Jacobian and $f$ is the $4 \times 1$ residual, is also discretized by using the 1-D form of the box method and central differences. The new solution is obtained by setting

$$
\left[\begin{array}{l}
n \\
v \\
T \\
\phi
\end{array}\right] \leftarrow\left[\begin{array}{l}
n \\
v \\
T \\
\phi
\end{array}\right]+t\left[\begin{array}{l}
\delta n \\
\delta v \\
\delta T \\
\delta \phi
\end{array}\right]
$$

where $t$ is a damping factor [12] between 0 and 1, chosen to insure that the norm of the residual $f$ decreases monotonically.

The operator Jacobian has the following block structure:

$$
J=\left[\begin{array}{cccc}
\frac{\delta f_{n}}{\delta n} & \frac{\delta f_{n}}{\delta v} & 0 & 0 \\
\frac{\delta f_{r}}{\delta n} & \frac{\delta f_{r}}{\delta v} & \frac{\delta f_{r}}{\delta T} & \frac{\delta f_{r}}{\delta \phi} \\
\frac{\delta f_{T}}{\delta n} & \frac{\delta f_{T}}{\delta v} & \frac{\delta f_{T}}{\delta T} & 0 \\
\frac{\delta f_{\phi}}{\delta n} & 0 & 0 & \frac{\delta f_{\phi}}{\delta \phi}
\end{array}\right]
$$

After discretization, each block is diagonal, bi-diagonal, or tri-diagonal. Newton's equation (24) may be solved directly by sparse matrix techniques, or by block iterative methods. 
As an initial guess for the solution, we take $n=N(x)$, $v=0, T=T_{0}$, and $e \phi=T \ln \left(n / n_{e}\right)$. This initial guess is very far from the hydrodynamic model solution even for $V=0.5$, so we use a continuation method in $V$ : i.e., we start with $V \ll 0.5$, compute the solution, increment $V$ by a small amount $\Delta V$, compute the new solution, etc. This method works well up to $V=10$ for the parameters discussed in this paper. At each stage, the Newton method converges quadratically when the variations of $\kappa, \tau_{p}$, and $\tau_{w}$ with respect to $n$ and $T$ are included. Typically between two and six Newton iterations are performed at each stage.

\section{Computations for Subsonic Flow}

Simulations of the ballistic diode using the hydrodynamic model were performed for biases $V=0.5,1,1.5$, and $2 \mathrm{~V}$. Fig. 1 shows the temperature $T(x)$ of the electron gas. Note that the temperature increases as the electrons flow from left to right across the $n$ region. The maximum temperature is an order of magnitude higher than ambient for $V=2 \mathrm{~V}$. Recall that the drift-diffusion model assumes the electron temperature $T=T_{0}$ throughout the device.

The temperature curves for $V=0.5,1$, and $1.5 \mathrm{~V}$ agree almost exactly with those of [11], even though we used 60 equal grid intervals and central differences, while $\mathrm{Ru}-$ dan, Odeh, and White used 30 or 100 interior grid points (with more points near the $n^{+}-n$ boundaries) and a Scharfetter-Gummel discretization.

Since $n$ and $T$ may exhibit strong variations over small regions, the Scharfetter-Gummel discretization (with its exponential fitting over each element) does resolve the hydrodynamic solution somewhat more efficiently for subsonic flow than the central difference discretization with equidistant grid points. However, even for subsonic flow, we expect the central difference discretization plus automatic mesh refinement [16] to be competitive with the Scharfetter-Gummel approach. For cases where the electron flow can become supersonic, a hyperbolic method (upwind, Lax-Wendroff, or Godunov) designed to resolve solution discontinuities (shocks and contacts) is appropriate.

In Fig. 2 we plot the electron velocity $v(x)$. Note the velocity overshoot phenomenon $v>v_{s}$ throughout most of the $n$ region. These curves do differ from those of [11], since there the authors took $\kappa_{0}=5 / 2$ in (18) for $\kappa$ instead of the value $\kappa_{0}=3 / 2$ which they currently recommend. The new value leads to a quantitative shift up in the velocity curves in Fig. 2, but leaves the temperature curves in Fig. 1 basically unchanged. (If we use the factor $5 / 2$, we recover the velocity curves of [11].)

Fig. 3 displays the Mach number $v(x) / c$ of the electrons. Typical Mach numbers for these flows are around 0.5 to 0.7 . As a self-consistency check for our elliptic numerical methods, we note that the flows are everywhere subsonic. (The one value of $v / c$ for $V=1$ which lies above Mach 1 is a grid effect. If more points are placed near the right $n-n^{+}$boundary, $v / c$ falls below 1.)

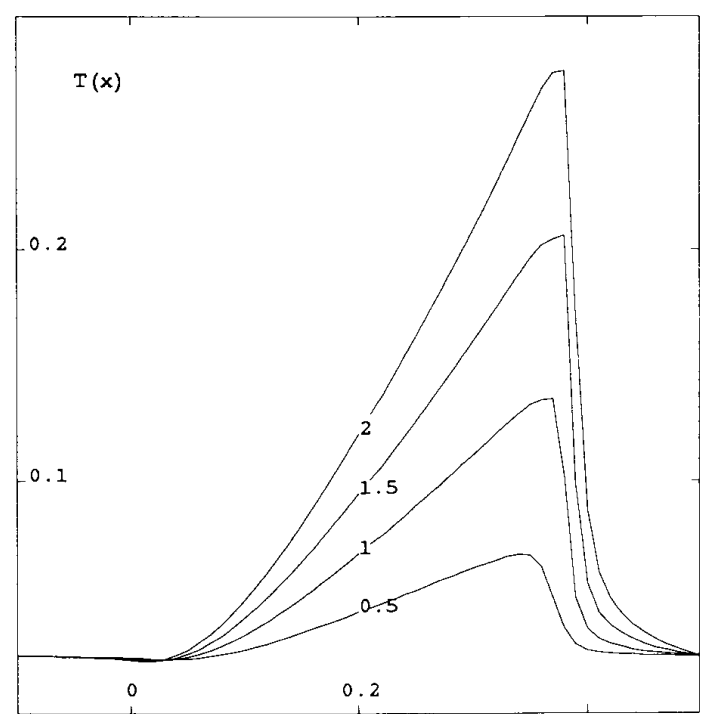

Fig. 1. Electron temperature $T(x)$ in electron volts for $V=0.5,1,1.5$, and 2 V. $x$ is in microns.

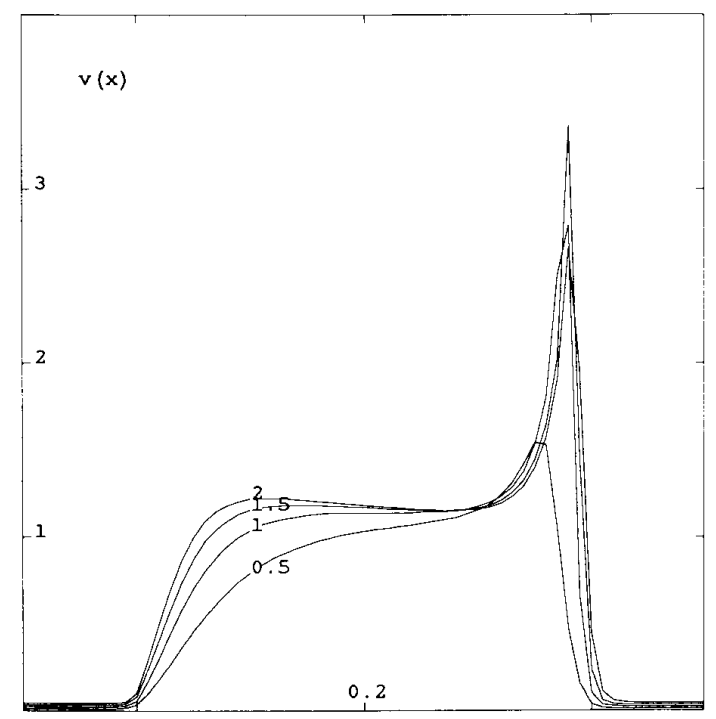

Fig. 2. Electron velocity $v(x)$ in $10^{7} \mathrm{~cm} / \mathrm{s}$ for $V=0.5,1,1.5$, and $2 \mathrm{~V}$. $x$ is in microns.

\section{Block Iterative Methods}

The 1-D ballistic diode serves as a numerical laboratory for investigating methods which will be essential for computational efficiency in 2-D problems.

The form of (26) for the operator Jacobian suggests [15] solving Newton's equation (24) by the iterative GaussSeidel splitting. Rewrite (24) and (25) as

$$
J_{k} x=-f_{k} ; \quad z_{k+1}=z_{k}+t_{k} x
$$

where $x=(\delta n, \delta v, \delta T, \delta \phi)^{T}, z=(n, v, T, \phi)^{T}$, and $k=$ $1,2, \cdots$ labels the outer Newton iterations. Then the 


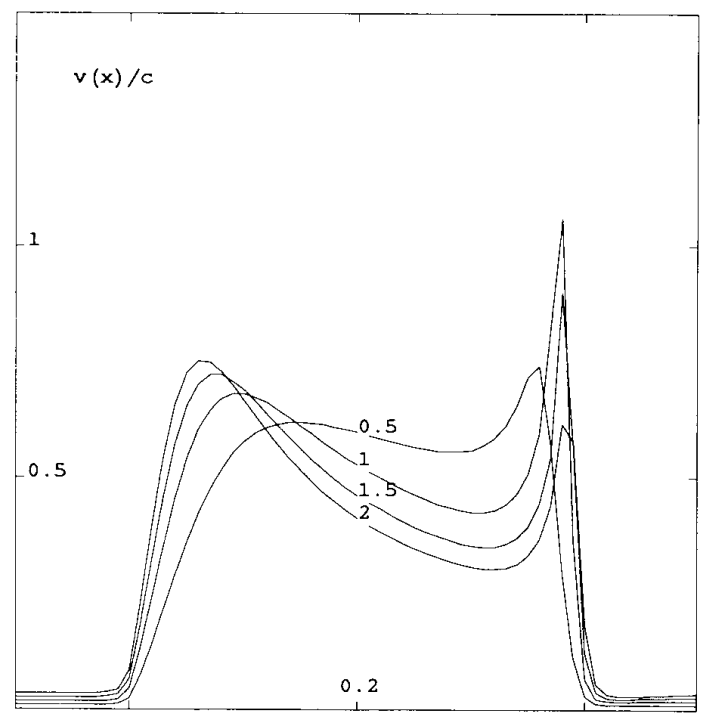

Fig. 3. Electron Mach number $v(x) / c$ for $V=0.5,1,1.5$, and $2 \mathrm{~V} . x$ is in microns.

iterative Gauss-Seidel splitting is

$$
L_{k}\left(x_{n}-x_{n-1}\right)=-\left(J_{k} x_{n}+f_{k}\right)
$$

where $L_{k}$ is the lower triangular part of $J$ and $n=1,2$, $\cdots, N_{\mathrm{GS}}$ labels the inner Gauss-Seidel iterations. After discretization, (28) may be viewed as a block iterative method for the discretized block system (27).

In one dimension, our computational experience indicates that solving the discrete version of Newton's equation by sparse matrix methods is faster than using block iterative methods. However, in two dimensions, block iterative methods will be essential as the number of PDE's increases. Block iterative methods are ideally suited for the hydrodynamic model, since in 2-D the number of PDE's is $K=4 N_{\text {species }}+1$, where $N_{\text {species }}$ may be as large as four in GaAs (upper and lower valley electrons and holes).

In more detail, solving $K$ PDE's on an $N \times N$ grid by sparse direct methods requires $O\left(K^{3} N^{3}\right)$ operations, while block iterative methods require $O\left(K N^{3}\right)+N_{\mathrm{GS}} \cdot O\left(K N^{2}\right.$ In $(N))$ operations as $N$ and $K$ become large. The crossover from sparse direct to block iterative methods becomes favorable if $N_{\mathrm{GS}} \ll K^{2} N / \ln (N)$.

The number of inner iterative loops (versus outer Newton loops) is optimized by using the methods of [12]. We define

$$
\alpha_{k, n}=\left\|f_{k}+J_{k} x_{n}\right\|\left\|f_{k}\right\|^{-1} .
$$

If the Gauss-Seidel iteration converges, $\alpha_{k, n} \rightarrow 0$ as $n \rightarrow$ $\infty$. We stop the inner iterations when

$$
\alpha_{k, n} \leq \alpha_{0}\left\|f_{k}\right\| /\left\|f_{0}\right\|
$$

where $\alpha_{0} \in(0,1)$.

Computational experiments for a 2-D Si MESFET will be presented in [17].

\section{Mathematical Analysis of the Hydrodynamic EquaTions}

\section{Steady-State Problem}

The specification of a subsonic physical regime for the carrier velocity, i.e., $|\boldsymbol{v}|<\sqrt{T / m}$, provides certain mathematical simplifications, in that the derivative multiplier matrix, for the linearized system, is positive definite and symmetric in this case. This justifies the characterization of the system as elliptic. There is, however, a complication. Simply because the system at the solution behaves subsonically, it does not follow that iterates far from the solution will necessarily satisfy a comparable inequality. A local analysis then is forced to assume either the existence of a solution with this property, and to restrict approximate solutions to be sufficiently close in graph and slope to maintain this, or to assume the equivalent, via the Newton-Kantorovich theorem, in terms of negligible residuals and subsonic properties. Because the Newton-Kantorovich theorem expresses both an existential as well as an approximation result, we use this as the motivational basis for our approach. Thus we begin by introducing the linearized problem at a subsonic point in function space, and proceed to discuss conditions under which the linear increments are bounded in appropriate norms. This is equivalent to the well-known property of an operator Newton method, which uniformly bounds the operator derivative inverse maps. General operator Newton methods with this property, and Lipschitz derivative regularity, have been studied in [12] and [18]. In addition, continuation, clearly relevant here, is discussed in these references.

The linearized steady-state hydrodynamic equations in 1-D assume the following form ( symmetry considerations dictate the interchange of $n$ and $v$ ):

$$
\begin{gathered}
{\left[\begin{array}{c}
0 \\
0 \\
-\frac{1}{n} \frac{d}{d x}\left(\kappa \frac{d \delta T}{d x}\right) \\
\epsilon \frac{d^{2} \delta \phi}{d x^{2}}
\end{array}\right]+\left[\begin{array}{ll}
A & B \\
C & D
\end{array}\right] \frac{d}{d x}\left[\begin{array}{l}
\delta v \\
\delta n \\
\delta T \\
\delta \phi
\end{array}\right]} \\
+\left[\begin{array}{ll}
E & F \\
G & H
\end{array}\right]\left[\begin{array}{c}
\delta v \\
\delta n \\
\delta T \\
\delta \phi
\end{array}\right]=f .
\end{gathered}
$$

The (spatially dependent) eigenvalues of the symmetric matrix $A$ are calculated to be

$$
\lambda=\frac{1}{2}\left(n+\frac{T}{m n}\right) \pm \frac{1}{2} \sqrt{\left(n+\frac{T}{m n}\right)^{2}-4\left(\frac{T}{m}-v^{2}\right)}
$$


The smaller eigenvalue is clearly positive at a subsonic point in dependent variable space, i.e., a point for which $n$ and $T$ are strictly positive, and $v^{2}<T / m$.

It is possible to obtain estimates for the linearized equation

$$
F^{\prime}(v, n, T, \phi)[u, w]=f=\left[f_{1}, f_{2}\right]
$$

where $u=[\delta v, \delta n]$ and $w=[\delta T, \delta \phi]$. Here, $F^{\prime}$ denotes the left-hand side of (31). By taking the inner product of (33) with $[d u / d x, 0]$, we obtain the relation (written in terms of vector $L^{2}$ inner products)

$$
\begin{aligned}
& \left(A u^{\prime}, u^{\prime}\right)+\left(B w^{\prime}, u^{\prime}\right)+\left(E u, u^{\prime}\right)+\left(F w, u^{\prime}\right) \\
& =\left(f_{1}, u^{\prime}\right) .
\end{aligned}
$$

By the positive-definiteness of $A$ (assumed uniform here), we conclude that

$$
\begin{aligned}
\left\|u^{\prime}\right\|^{2} \leq & \operatorname{const}\left(A u^{\prime}, u^{\prime}\right) \leq \text { const }\left\{\|B\|\left\|w^{\prime}\right\|\left\|u^{\prime}\right\|\right. \\
& +\|E\|\|u\|\left\|u^{\prime}\right\|+\|F\|\|w\|\left\|u^{\prime}\right\| \\
& \left.+\left\|f_{1}\right\|\left\|u^{\prime}\right\|\right\}
\end{aligned}
$$

where $\|\cdot\|$ denotes the $L^{2}$ norm. Assuming for the moment that upper bounds exist for $|d n / d x|,|d v / d x|$, and $T$, and a lower bound for $n$ exists, we deduce from (35) that $\left\|u^{\prime}\right\|$ is bounded in terms of $\|u\|,\left\|f_{1}\right\|$, and the $H^{1}$ norm of $w,\|w\|_{H^{1}}=\sqrt{\|w\|^{2}+\left\|w^{\prime}\right\|^{2}}$.

At a solution point, bounds for $|d n / d x|$ and $|d v / d x|$ are obtained by using (20):

$$
\begin{aligned}
& \left|\frac{d n}{d x}\right| \leq\left(\frac{T}{m}-v^{2}\right)^{-1}\left(\frac{|n v|}{\tau_{p}}+\frac{n}{m}\left|\frac{d T}{d x}-e \frac{d \phi}{d x}\right|\right) \\
& \left|\frac{d v}{d x}\right| \leq|v|^{3}\left(\frac{T}{m}-v^{2}\right)^{-1}\left(\frac{|v|}{\tau_{p}}+\frac{1}{m}\left|\frac{d T}{d x}-e \frac{d \phi}{d x}\right|\right) .
\end{aligned}
$$

Bounds (36) and (37) can then serve as a starting point to obtain derivative bounds for functions "close by" in a local theory.

The elimination of $\|u\|$ in the estimation of $\left\|u^{\prime}\right\|$ is more subtle, and focuses attention on an additional property of the subsonic hydrodynamic system necessary to derive a Newton-Kantorovich theory. This property, not to be confused with the positive-definite property of the matrix $A$, may properly be termed a coerciveness property.

Specifically, define the operator $J$ by

$$
J u=A u^{\prime}+E u .
$$

We require, for $u$-coerciveness, that

$$
(J u, u) \geq \omega_{0}(u, u)
$$

for some positive constant $\omega_{0}$. The adjoint operator is defined by

$$
J * u=-\frac{d}{d x}(A u)+E^{*} u
$$

so that

$$
\begin{aligned}
(J u, u) & =\frac{1}{2}\left((J u, u)+\left(J^{*} u, u\right)\right) \\
& =\left(\frac{1}{2}\left(E+E^{*}-\frac{d A}{d x}\right) u, u\right) .
\end{aligned}
$$

The property of $u$-coerciveness reduces to the assumption that $M=E+E^{*}-d A / d x$ has positive eigenvalues, uniformly bounded away from zero.

By taking the inner product of (33) with $[u, 0]$, we obtain the relation

$$
(J u, u)+\left(B w^{\prime}, u\right)+(F w, u)=\left(f_{1}, u\right)
$$

so that

$$
\begin{aligned}
\|u\|^{2} \leq & \frac{1}{\omega_{0}}\left\{\left\|f_{1}\right\|\|u\|+\|B\|\left\|w^{\prime}\right\|\|u\|\right. \\
& +\|F\|\|w\|\|u\|\} .
\end{aligned}
$$

It follows that $\|u\|$ can be estimated in terms of the $H^{1}$ norm of $w$ and in terms of $\left\|f_{1}\right\|$. By using (35) and (43), we conclude that

$$
\|u\|^{2}+\left\|u^{\prime}\right\|^{2} \leq \text { const }\left\{\|w\|^{2}+\left\|w^{\prime}\right\|^{2}+\left\|f_{1}\right\|^{2}\right\}
$$

provided the subsonic and $u$-coerciveness properties are assumed at the solution, where the linearization is carried out locally, near the solution in an appropriate sense.

In order to obtain an inequality of the form

$$
\begin{aligned}
& \|u\|^{2}+\left\|u^{\prime}\right\|^{2}+\|w\|^{2}+\left\|w^{\prime}\right\|^{2}+\left\|w^{\prime \prime}\right\|^{2} \\
& \quad \leq \text { const }\|f\|^{2},
\end{aligned}
$$

which is an appropriate statement of the uniform derivative invertibility required for the Kantorovich approach, one requires a parallel concept of $w$-coerciveness. In this context, this simply means that the coefficient of $\delta T$ in the linearized version of (21), i.e., the nonzero entry of $H$ in (31), is sufficiently positive in a uniform sense. When this holds, estimation can be made by taking the inner product of (33) with $[0, w]$. We find that

$$
\|w\|_{H^{1}}^{2} \leq \text { const }\left\{\|u\|^{2}+\left\|u^{\prime}\right\|^{2}+\left\|f_{2}\right\|^{2}\right\} \text {. }
$$

By (44) and further use of the $w$-coerciveness property, we conclude that

$$
\|u\|_{H^{1}}^{2}+\|w\|_{H^{1}}^{2} \leq \text { const }\|f\|^{2} .
$$

The sharpening of (47) to (45) can be made by direct estimation of second derivatives within the linearized system.

The following result follows from the preceding estimates, and from the Newton-Kantorovich theory. It assumes a Lipschitz continuous derivative $F^{\prime}$.

Proposition: If a function space localization is possible, with uniformly subsonic properties for the approximations, and uniform coerciveness in the linearized systems, then Newton's method, with a sufficiently small 
initial residual, converges $\mathrm{R}$-quadratically to a solution of the hydrodynamic equations.

\section{Time-Dependent Problem}

Space limitations prevent us from providing an analogous discussion of the evolution system. A few brief comments are in order, however. If one thinks of the appropriate linearized evolution system as discretized in time, then the $u$ - and $w$-coerciveness properties may be expected to hold for $\Delta t$ sufficiently small. In this case, the matrix multiplier of $u$ is not longer symmetric, but the eigenvalues are $v \pm \sqrt{T / m}$, so that the corresponding subsystem is symmetrizable. The appropriate boundary condition for the 1-D linearized evolution system may be specified by the Friedrichs theory of admissible boundary conditions [19].

\section{Boundary Conditions}

For general electron flows in one dimension, boundary conditions for both steady-state and time-dependent problems may be determined by applying Friedrichs' theory [19]. Boundary conditions for two- and three-dimensional problems may be specified by applying the theories of Agemi [20], Oliger and Sundstrom [21], and Osher and Chakravarthy [22]. These theories apply to the Euler equations, and must be modified in light of the fact that in the hydrodynamic model one of the hyperbolic modes of the Euler equations has become a parabolic mode.

\section{REFERENCES}

[1] K. Blotekjaer, "Transport equations for electrons in two-valley semiconductors," IEEE Trans. Electron Devices, vol. ED-17, pp. 38-47, 1970.

[2] M. Rudan and F. Odeh, "Multi-dimensional discretization scheme for the hydrodynamic model of semiconductor devices," COMPEL 5 ,pp. $149-183,1986$.

[3] R. K. Cook and J. Frey, "Two-dimensional numerical simulation of energy transport effects in Si and GaAs MESFET's," IEEE Trans. Electron Devices, vol. ED-29, pp. 970-977, 1982.

[4] _ "An efficient technique for two-dimensional simulation of velocity overshoot effects in Si and GaAs devices, " COMPEL 1, pp. $65-871982$.

[5] G. Baccarani and M. R. Wordeman, "An investigation of steadystate velocity overshoot effects in Si and GaAs devices," Solid State Electron., 28, 407-416, 1985

[6] M. S. Shur and E. F. Eastman, "Ballistic transport in semiconductor at low temperatures for low-power high-speed logic,' IEEE Trans. Electron Devices, vol. ED-26, pp. 1677-1683, 1979.

[7] M. Heilblum, M. I. Nathan, D. C. Thomas, and C. M. Knoedler, "Direct observation of ballistic transport in GaAs," Phys. Rev. Lett., vol. 55 , pp. $2200-2203,1985$.

[8] T. E. Bell, "The quest for ballistic action," IEEE Spectrum, vol. 23, pp. $36-38,1986$.

[9] For an introduction to the Euler equations and shock waves, see $R$. Courant and K. O. Friedrichs, Supersonic Flow and Shock Waves. New York: Springer-Verlag, 1948

[10] C. L. Gardner, "Nonlinear hyperbolic waves in the hydrodynamic model for semiconductor devices, " to appear.

[11] F. Odeh, M. Rudan, and J. White, "Numerical solution of the hy drodynamic model for a one-dimensional semiconductor device," COMPEL 6, pp. 151-170, 1987

[12] R. E. Bank and D. J. Rose, "Global approximate Newton methods," Numer. Math., vol. 37, pp. 279-295, 1981.

[13] P. D. Lax, "Hyperbolic systems of conservation laws and the mathematical theory of shock waves," SIAM, Philadelphia, PA, 1972.

[14] M. V. Fischetti and M. Rudan, "Hydrodynamic and Monte Carlo simulation of an $n^{+}-n-n^{+}$submicron device," IEEE Trans. Electron Devices to be published.

[15] R. E. Bank, D. J. Rose, and W. Fichtner, "Numerical methods for semiconductor device simulation," SIAM J. Sci. Stat. Comput., vol. 4, pp. 416-435, 1983.

[16] M. J. Berger, "Adaptive finite difference methods in fluid dynamics," to appear.

[17] C. L. Gardner and D. J. Rose, "Nonlinear block iterative methods for the hydrodynamic semiconductor device model," to appear.

[18] J. W. Jerome, "Approximate Newton methods and homotopy for stationary operator equations," Constr. Approx. vol. 1, pp. 271-285, 1985

[19] K. O. Friedrichs, "Symmetric positive linear differential equations," Comm. Pure Appl. Math., vol. 11, pp. 333-418, 1958.

[20] R. Agemi, "Mixed problems for the linearized shallow water equations," Comm. Partial Diff. Eqs., vol. 5, pp. 645-681, 1980.

[21] J. Oliger and A. Sundstrom, "Theoretical and physical aspects of some initial value problems in fluid dynamics," SIAM J. Appl. Math. vol. 35, pp. 419-446, 1978.

[22] S. Osher and S. Charkravarthy, "Upwind schemes and boundary conditions with applications to Euler equations in general geometries,' J. Comp. Phys., vol. 50, pp. 447-481, 1983.

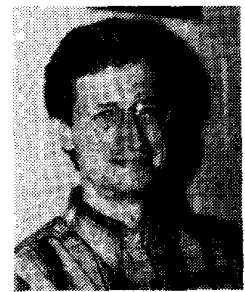

Carl L. Gardner received the B.A. degree from Duke University in 1973 and the Ph.D. degree from MIT in 1981 .

$\mathrm{He}$ is currently an Assistant Professor of Computer Science and Mathematics at Duke University. Previously he was an Associate Research Scientist at the Courant Institute, Assistant Professor of Physics at Bowdoin College, and Instructor of Physics at MIT. His current research interests lie in semiconductor device and process simulations, and computational granular and fluid

dynamics.

Dr. Gardner is a member of SIAM and the American Physical Society.

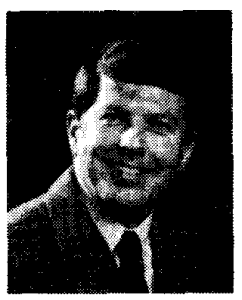

Joseph W. Jerome received the Ph.D. degree from Purdue University, Lafayette, IN, in 1966.

He has been Professor of Mathematics and Applied Mathematics at Northwestern University, Evanston. since 1976. He has previously held factulty positions at the University of Wisconsin. Madison, and Case Western Reserve University. and has held visiting positions at Oxford University, England, the University of Texas, Austin. Bell Laboratories, Murray Hill, NJ, and the University of Chicago, Chicago, IL. His current research interest is in nonlinear analysis, focusing on applied mathematics, numerical analysis, and partial differential equations. These interests have merged in the study of VLSI models. His most recent book is Approximation of Nonlinear Evolution Systems, New York, Academic Press, 1983. He was British Science Council Senior Visiting Fellow at Oxford in 1974. 1975, and is a former National Science Foundation Graduate Fellow.

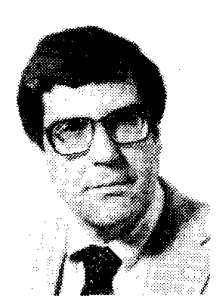

Donald J. Rose received the B.A. degree in mathematics from the University of California at Berkeley in 1966, and the M.A. and Ph.D. degrees in applied mathematics from Harvard University, Cambridge, MA. in 1967, and 1970, respectively.

From 1970 to 1984 he held research positions at the University of Denver, Harvard University. Vanderbilt University, and AT\&T Bell Laboratories. In 1984, he became the Chairman of the Computer Science Department at Duke University, Durham, NC, where he investigates problems involving numerical analysis and scientific computation.

Dr. Rose is a member of SIAM, AMS, MAA, and ACM. 